\title{
Chaotic Phenomena in a Simple 3D Autonomous Circuit with a Diode Pair
}

\author{
G. KANDIBAN ${ }^{a}$, S. MANIMARAN ${ }^{b}$ AND A.V. BALACHANDRAN ${ }^{c, *}$ \\ ${ }^{a}$ Department of Physics, Thanthai Hans Roever College, Perambalur - 621 212, India \\ ${ }^{b}$ Department of Physics, Roever Engineering College, Perambalur - 621 212, India \\ ${ }^{c}$ Department of Physics, A.A. Government Arts College, Musiri, Tiruchirappalli - 621 201, India
}

(Received April 6, 2011; revised version August 5, 2011; in final form November 18, 2011)

The simple 3D-chaotic dynamics of an autonomous oscillator circuit was studied by measuring its responsible in the form of phase-portrait, power spectrum and chaotic time series. The new realization combines attractive features of the current feedback op-amp operating in both voltage and current modes to construct the active linear negative conductance. The component count is reduced and the chaotic spectrum is extended to higher frequencies. In addition, a buffered and isolated voltage output directly representing a state variable is made available. The circuit consists of just three linear elements (two capacitors and one inductor), one linear negative conductance and two ideal diodes. The power spectra are presented to confirm the strong chaotic nature of the oscillations of the circuit. The performance of the circuit is investigated by means of experimental simulation and numerical confirmation of the appropriate differential equations. The features of the obtained results are respected for various engineering systems such as chaos communication systems with robustness against various interferences.

PACS: 05.45.-a, 05.45.Xt, 05.45.Ac

\section{Introduction}

In the present report the behavior of a third-order autonomous oscillator circuit has been studied. This circuit consists of two active elements, one linear negative conductance and one cubic nonlinearity exhibiting symmetrical piecewise-linear $v-i$ characteristics, two linear capacitances $\left(C_{1}\right.$ and $\left.C_{2}\right)$ and one linear inductor $(L)$ are also included in the circuit, serve as the control parameters [1-5].

Most chaotic and bifurcation effect cited in the literature have been observed in electrical circuits. They include the period-doubling route to chaos [6], the intermittency route to chaos [7], and the quasi-periodicity route to chaos and of course the crisis [8-10]. This popularity is attributed to the advantages which electric circuits offer to experimental chaos studies, such as robustness and convenient implementation.

In this work we introduce an attractive combined voltage-current capabilities of a current feedback op-amp (CFOA) which are used to synthesize linear negative conductance. In addition, a buffered and isolated voltage output that directly represents a state variable is made available while the operating frequency is extended. The simple-3D autonomous third-order oscillator circuit realizes period-doubling route to chaos followed by periodic window and then to strong chaos through boundary crisis etc. We consider that such complicated chaotic time

\footnotetext{
* corresponding author; e-mail: brsbala@rediffmail.com
}

waveforms are expected to be utilized for realization of several chaotic applications such as chaos communication system with robustness against various interferences including multi user access.

\section{Experimental realization}

The experimental realization of the third-order autonomous oscillator and symmetrical cubic nonlinear characteristics are shown in Fig. 1a,b respectively $[11,12]$. The characteristics of the negative conductance are mathematically represented by $i=-G_{1} V_{1}$.

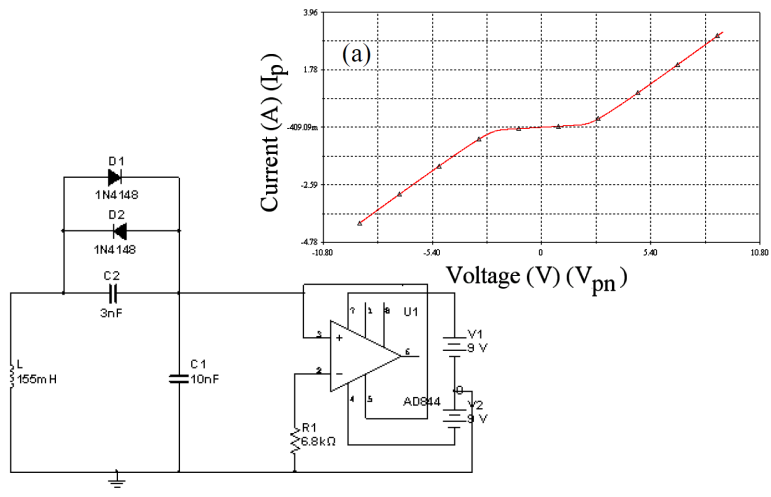

Fig. 1. Simple-3D autonomous oscillator circuit. (a) Symmetrical cubic nonlinear characteristics.

Figure 1 illustrates a circuit diagram with which the authors deal in this paper, where $L, C_{1}, C_{2}$, and $-\frac{1}{R_{1}}=$ 
$-G_{1}$ are an inductor, capacitors, and a linear negative conductance, respectively. The symmetrical cubic nonlinearity is designed with the help of two signal diodes. The $v-i$ characteristics of the global nonlinearity which can therefore be approximated by a cubic function of the form with $a<0$ and $b>0$ :

$$
f(V)=v+a V+b V^{3} .
$$

The constant term $v$ describes the input current offset of the op-amp which can be practically adjusted to zero using the potentiometer $(v=0)$. By applying Kirchhoff's laws to the equivalent circuit of Fig. 1 we obtain the following set of differential equations:

$$
\begin{aligned}
& C_{1} \frac{\mathrm{d} V_{1}}{\mathrm{~d} t}=-\left(G_{1} V_{1}+i_{L}\right), \\
& C_{2} \frac{\mathrm{d} V_{2}}{\mathrm{~d} t}=i_{L}-i_{N}, \\
& L \frac{\mathrm{d} i_{L}}{\mathrm{~d} t}=V_{1}-V_{2} .
\end{aligned}
$$

While $V_{1}$ and $V_{2}$ are the voltages across the capacitors $C_{1}$ and $C_{2}$, and $i_{L}$ denotes the current through the inductance $(L)$, respectively, the term $i_{N}$ representing the characteristics of the symmetrical cubic nonlinearity can be expressed mathematically as

$$
i_{N}=f\left(V_{2}\right)=a V_{2}+b V_{2}^{3} \text {. }
$$

The simple-3D autonomous oscillator circuit is also truly the Van der Pol-Duffing oscillator. This is because there is no locally active resistance $(R)$ in this circuit, only varying the inductance $(L)$ value, this circuit exhibits very interesting dynamical phenomena like period-doubling bifurcation sequence leading to chaos, period-doubling window and then to strong chaos through boundary condition $[13,14]$. However, in the parameter regimes investigated, important features like period-doubling window, strong chaos have not been reported.

For our present experimental study we have chosen the following typical values of the circuit in Fig. 1: $C_{1}=10 \mathrm{nF}, C_{2}=3 \mathrm{nF}$. The negative conductance $G_{1}=-0.14705 \mathrm{mS}$ and cubic nonlinearity $a<0$ and $b>0$. Here the variable inductor $(L)$ is assumed to be the control parameter.

By increasing the value of $L$ from $100 \mathrm{mH}$ to $250 \mathrm{mH}$, the circuit behavior of Fig. 1 is found to transmit from a period doubling route to chaos, and then to period doubling window through strong chaos followed by boundary crisis etc. From our experimental investigations, we find that for the value of $L$ above $100 \mathrm{mH}$, limit cycle motion is obtained, when the value of $L$ is increased, particularly in the range $L=210 \mathrm{mH}$ the system displays a double band chaotic motion. The projection of the attractors of the $V_{1}-V_{2}$ and current sensing resistor with voltage plane of cathode ray oscilloscope are shown in Fig. 2 for various values of control parameter $L$. Figure 3 shows the experimental chaotic time series which were registered using a cathode ray oscilloscope for discrete values of $L$ serving as the control parameter.
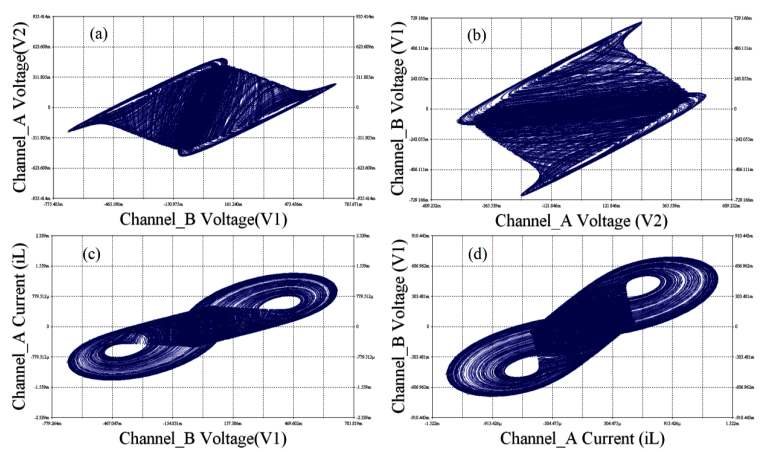

Fig. 2. Typical experimental phase portraits of the system corresponding to different regimes.

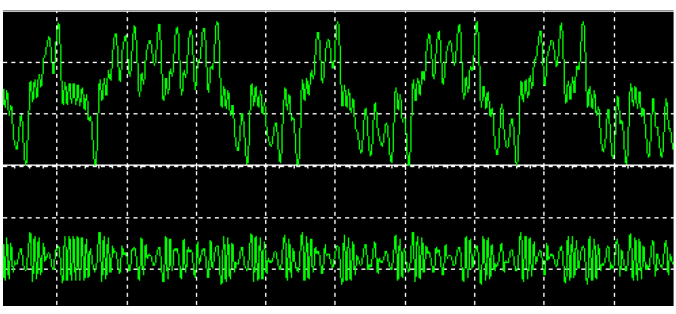

Fig. 3. Time-domain measurements of the proposed autonomous oscillator.

The third-order autonomous oscillator circuit with the symmetrical cubic nonlinearity can produce strong chaos seen in Fig. 4 from which we observe clearly that there are large broad-band power spectra. The distribution of power in a signal $x(t)$ is the most commonly quantified by means of the power density spectrum or simply power spectrum. It is the magnitude-square of the Fourier transforms of the signal $x(t)$. It can detect the presence of chaos when the spectrum is broad-banded. The power spectrum corresponding to the voltages $V_{1}(t)$ and $V_{2}(t)$ waveforms across the capacitors $C_{1}$ and $C_{2}$ for the chaotic regimes are shown in Fig. 4 which resembles broad-band spectrum noise.

\section{Numerical realization}

For a convenient numerical analysis of the experimental systems given by Eqs. (1.1), (1.2), we rescale the

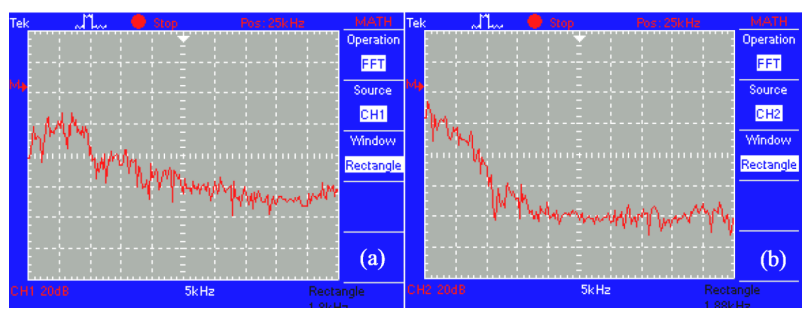

Fig. 4. Power spectrum of the signals (a) $V_{1}(t)$ and (b) $V_{2}(t)$ from the circuit of autonomous oscillator. 
parameters as $V_{1}=V x_{1}, V_{2}=V x_{2}, i_{L}=\sqrt{\frac{C_{1}}{L}} V x_{3}$, $t=\sqrt{L C_{1}} \tau, v=\frac{C_{1}}{C_{2}}, \gamma=G_{1} \sqrt{\frac{L}{C_{1}}}, \alpha=a \sqrt{\frac{L}{C_{1}}}$, $\beta=b \sqrt{\frac{L}{C_{1}}}$, and then redefine $\tau$ as $t$. Then the normalized equations of the third-order autonomous oscillator circuit (Fig. 1) are

$$
\begin{aligned}
& x_{1}=-\left(\gamma x_{1}+x_{3}\right), \\
& x_{2}=v\left(x_{3}-\alpha x_{2}-\beta x_{2}^{3}\right), \\
& x_{3}=\left(x_{1}-x_{2}\right),
\end{aligned}
$$

where

$$
f\left(x_{2}\right)=\alpha x_{2}+\beta x_{2}^{3} .
$$

The dynamics of Eqs. (2.1), (2.2) now depends on the parameters $\alpha, \beta, \gamma, v$ and $V=1$. The experimental results have been verified by computer simulation of the normalized Eqs. (2.1), (2.2) using the standard fourth-order Runge-Kutta method for a specific choice of system parameters employed in the laboratory experiments.

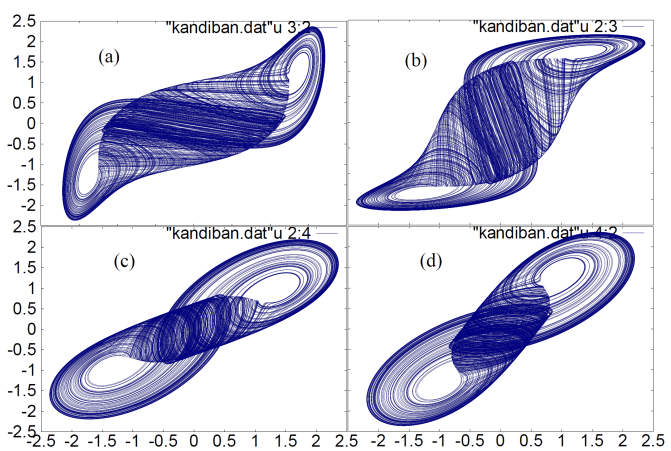

Fig. 5. Chaotic region observed from the circuit of Fig. 1. Projection onto (a) (), (b) $\left(V_{1}, V_{2}\right),(\mathrm{c})(\mathrm{,})$, and $(\mathrm{d})($, ) plane.

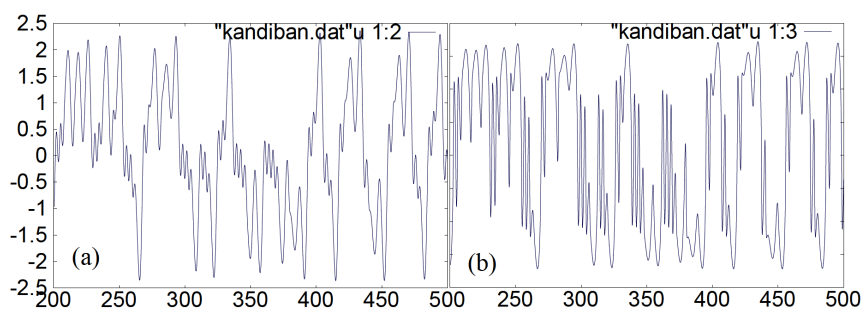

Fig. 6. Time waveform for $L_{1}=210 \mathrm{mH}$ for (a) $V_{1}$ and (b) $V_{2}$.

That is, in the actual experimental setup the inductor $L$ is varied from $L=100 \mathrm{mH}$ up to $250 \mathrm{mH}$. Therefore in the numerical simulation we study the corresponding Eqs. (2.1), (2.2) for $L$ in the range $L=$ $(100 \mathrm{mH}, 250 \mathrm{mH})$. From our numerical investigations, we find that for the value of $L$ above $100 \mathrm{mH}$, limit cycle motion is obtained, when the value of $L$ is increased, particularly in the range $L=210 \mathrm{mH}$ the system displays a double band chaotic motion and then to period-doubling window through strong chaos followed by boundary crisis etc. These numerical results are summarized in the phase portraits given in the $\left(x_{1}-x_{2}\right)$, and $\left(x_{2}-x_{3}\right)$ planes which are shown in Fig. 5. Figure 6 shows that the numerical chaotic time series was registered using a discrete value of " $L$ " serving as the control parameter. It is gratifying to note that the numerical results agree qualitatively very well with that of the laboratory experiments.

\section{Conclusions}

It appears that the autonomous oscillator circuit presented in this paper is one of the simplest third-order systems reported so far. The circuit provides a higher bandwidth of chaotic signal with buffered output and functionally was demonstrated using a commercial CFOA. Its simplicity arises from the fact that (i) the negative conductance is a simple current feedback op-amp impedance converter. (ii) The symmetrical cubic nonlinearity is synthesized from two signal diodes. (iii) The circuit equations are the most simple because there is no locally active resistor $(R)$ in the circuit, where the inductance $(L)$ is the control parameter. The attractive features of this circuit are the presence of period-doubling route to chaos, period-doubling window through strong chaos followed by boundary condition etc. It is of further interest to study these aspects also in this system as well as the intermittency route to chaos and synchronization of coupled chaotic circuits of the present system for improved high security communication systems.

\section{References}

[1] T. Matsumoto, IEEE Trans. Circuits Syst. I 31, 1055 (1984).

[2] G.Q. Zhong, F. Ayrom, IEEE Trans. Circuits Syst. I 32, 501 (1985).

[3] M.P. Kennedy, IEEE Trans. Circuits Syst. I 41, 771 (1994).

[4] A. Namajunas, A. Tamasevicius, Electron. Lett. 32, 945 (1996).

[5] A. Namajunas, A. Tamasevicius, Electron. Lett. 31 335 (1995).

[6] M.P. Kenndy, Frequency 46, 66 (1992).

[7] R. Barboza, Int. J. Bifurc. Chaos 18, 1151 (2008).

[8] A. Tamasevicius, A. Namajunas, A. Cenys, Electron. Lett. 32, 957 (1996).

[9] H.B. Fostin, P. Woafo, Chaos Solitons Fract. 24, 1363 (2005).

[10] C.C. Hwang, H.Y. Chow, Y.K. Wang, Physica D 92, 95 (1996).

[11] G.P. King, S.T. Gaito, Phys. Rev. E 46, 3093 (1992).

[12] K. Murali, M. Lakshmanan, Phys. Rev. E 45, 1624 (1993).

[13] R. Barboza, L.O. Chua, Int. J. Bifurc. Chaos 18, 943 (2008).

[14] V. Balachandran, G. Kandiban, Ind. J. Pure Appl. Phys. 47, 823 (2009). 